Originalien und Übersichtsarbeiten

P. Brandt

Robert Koch-Institut, Berlin

\title{
Prinzipien der Risikoerwägung zur ,'Grünen Gentechnik"
}

ie Frage, ob mit dem Einsatz der „Grünen Gentechnik” Gefahren für die Menschen und die Umwelt verbunden sind, kann sachgerecht nicht nach dem Muster der Risikoabwägung - also unter der Voraussetzung, dass es Risiken gibt - erfolgen, sondern muss mit der Risikoerwägung - also der Frage, ob es Risiken gibt - beginnen. Derartige Risikoerwägungen zum Umgang mit gentechnisch veränderten Pflanzen dürfen sich nicht nur auf diese selbst beschränken, sondern müssen auch diejenigen mit einbeziehen, welche für die aus transgenen Pflanzen hergestellten Futter- oder Lebensmittel relevant sein könnten.

Gibt es aber derzeit nicht drängendere Probleme, als sich ausgerechnet mit Risikoerwägungen zum Umgang mit gentechnisch veränderten Pflanzen zu befassen, zu einem Zeitpunkt, wo - wie in den Medien immer wieder berichtet wird - nahezu $80 \%$ der deutschen Bevölkerung gentechnisch veränderte Lebensmittel ablehnen und diese im täglichen Leben derzeit auch keine bedeutende Rolle zu spielen scheinen. Stimmt aber diese Einschätzung der Bedeutung von gentechnisch veränderten Pflanzen?

\footnotetext{
Hinsichtlich Freisetzungsvorhaben mit gentechnisch veränderten Organismen liegt Deutschland in der EU mit nahezu 100 Vorhaben an siebenter Stelle. Weltweit gibt es mehr als 5000 Freisetzungsvorhaben.
}

Ein Maß für den Entwicklungsstand der „Grünen Gentechnik” auf dem Gebiet der Pflanzenzüchtung und damit u. a. auch auf dem Gebiet der späteren Futter- und Lebensmittelproduktion ist die Anzahl von Freisetzungsvorhaben mit gentechnisch veränderten Pflanzen wie auch die Anzahl von Genehmigungen für das Inverkehrbringen solcher transgener Pflanzen oder für aus ihnen hergestellten Futter- bzw. Lebensmittel. Von den weltweit mehr als 5000 Freisetzungsvorhaben mit gentechnisch veränderten Organismen entfallen auf die EU-Mitgliedsstaaten mehr als 1500 (Stand: Oktober 1999). Im EU-Vergleich liegt Deutschland mit nahezu 100 genehmigten Freisetzungsvorhaben auf dem siebenten Platz und damit zwar auf mittlerer Position, jedoch sollte nicht unbeachtet bleiben, daß rund $85 \%$ der Freisetzungen im Bereich der EU in den sechs EU-Mitgliedsstaaten Frankreich, Italien, England, Spanien, Niederlande und Belgien stattgefunden haben (Abb.1).

Für den Bereich der EU sind bislang acht uneingeschränkte sowie fünf eingeschränkte Genehmigungen für das Inverkehrbringen von gentechnisch veränderten Pflanzen erteilt worden (Tabelle 1).

In Anbetracht der außereuropäischen wirtschaftlichen Verflechtungen der EU-Mitgliedsstaaten sollte sich allerdings niemand von diesen niedrigen „europäischen” Zahlen darüber täu- schen lassen, dass weltweit bereits über 50 derartige Zulassungen für mehr als zehn verschiedene Kulturpflanzen vorliegen, die eine (oder mehrere) gentechnische Veränderung(en) besitzen [1]. Seit 1996 nimmt die Fläche für den kommerziellen Anbau dieser in Verkehr gebrachten, transgenen Kulturpflanzen stetig zu und hat im Jahr 1998 bereits eine Größe von 27,8 Mill. Hektar erreicht (Abb. 2); der Marktwert der 1998 in den Handel gebrachten transgenen Pflanzen betrug etwa 1,5 Milliarden US\$. Eine Risikoerwägung im Bereich der „Grünen Gentechnik” ist grundsätzlich von den drei Prämissen case-by-case, step-bystep und Stand der Wissenschaft abhängig, welche eigentlich selbstverständlich sind, in den Medien oder bei öffentlichen Diskussionen aber leider immer wieder außer Acht gelassen werden.

\section{Pauschalisierende Bewertungen sind für gentechnische Vorhaben unbrauch- bar, Risikoerwägungen müssen am je- weiligen konkreten Einzelfall vorge- nommen werden.}

*Gekürzte Fassung eines Vortrages vom 27.1.1999 im Robert Koch-Institut; statistische Angaben wurden aktualisiert.

Prof.Dr. Dr. Peter Brandt

Zentrum Gentechnologie, Robert Koch-Institut, Wollankstraße 15-17, 13187 Berlin 


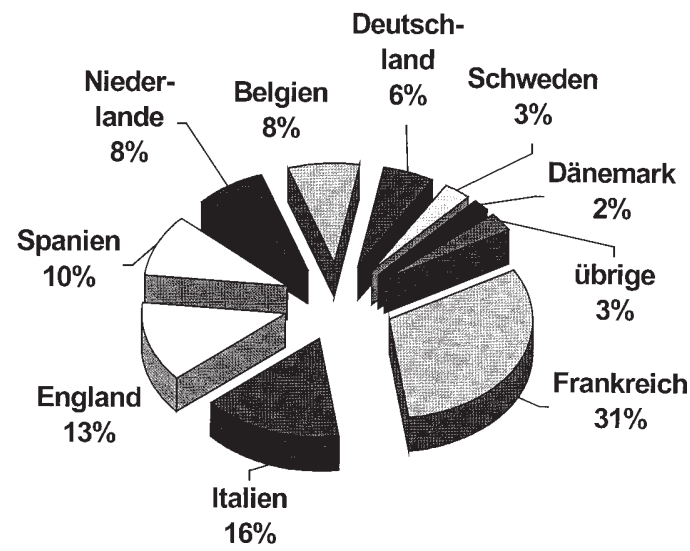

Abb. $1<$ Prozentuale Verteilung der angemeldeten Freisetzungen von gentechnisch veränderten Pflanzen im Europäischen Beteiligungsverfahren (SNIF = Summary Notification and Information Format) auf die beteiligten EU-Mitgliedsstaaten (übrige = Finnland, Griechenland, Portugal, Irland, Österreich) (Stand: Oktober 1998; Quelle: Robert KochInstitut, Berlin; http://www.rki.de)
Vergegenwärtigt man sich, dass mittels diverser, molekularbiologischer Methoden grundsätzlich jegliche Pflanzenspezies mit einem oder mehreren der verschiedenartigsten Genkonstrukte transformiert werden kann, wobei die einzelnen Komponenten dieser chimären Genkonstrukte wiederum aus ganz unterschiedlichen Spenderorganismen herrühren können [2], so ist es fast trivial, darauf hinzuweisen, dass pauschalisie- rende Bewertungen für gentechnische Belange unbrauchbar sind und eine Risikoerwägung nur im jeweils vorliegenden Einzelfall sachgerecht ist.

Solche Riskoerwägungen hängen aber nicht nur von den Eigenschaften der jeweiligen gentechnisch veränderten Pflanze ab, sondern auch von den Bedingungen, unter denen ihre Eigenschaften experimentell überprüft werden sollen. Sinnvollerweise wird man nach der Eta- blierung einer transgenen Pflanze sie zunächst im Laborbereich untersuchen und erst dann, wenn genügend Erfahrung mit dieser transgenen Pflanze vorliegt, zu Versuchen im Gewächshaus und dann zu solchen im Freiland übergehen (Abb.3).Vorhaben mit gentechnisch veränderten Pflanzen, die im Labor, im Gewächshaus oder - räumlich und zeitlich begrenzt - im Freiland stattfinden, haben experimentellen Charakter und sind auch unter dieser Prämisse zu bewerten. Erst mit dem Inverkehrbringen werden die räumlichen und zeitlichen Begrenzungen in der Regel verlassen, und der Experimentiercharakter liegt nicht mehr vor. In diesem mehrstufigen System begründet sich die schrittweise Aufhebung des Containments auf der Erfahrung, die man mit den transgenen Pflanzen in den jeweils zuvor durchgeführten Versuchen erworben hat. Diese erworbene Erfahrung ist auch ein Bestandteil der dritten Prämisse, dem Stand der Wissenschaft.

Zum Stand der Wissenschaft gehören alle derzeitigen wissenschaftlichen Erkenntnisse, nicht nur die in Kreisen

Tabelle 1

Produkte aus dem Bereich der „,Grünen Gentechnik", für welche ein Inverkehrbringen in der Europäischen Union genehmigt wurde

\begin{tabular}{|c|c|c|c|}
\hline Antragsteller & GVO & gentechnische Veränderung & Beschränkungen \\
\hline Seita & Tabak & Herbizid-Toleranz & - \\
\hline Plant Genetic Systems & $\operatorname{Raps}^{* *}$ & $\begin{array}{l}\text { Männliche Sterilität zur Erzeugung von Hybridsaatgut } \\
\text { sowie Herbizid-Toleranz }\end{array}$ & nur zu Züchtungszwecken \\
\hline Ciba Geigy & Mais & Insekten-Resistenz und Herbizid-Toleranz & - \\
\hline Bejo Zaden BV & Radicchio & Männliche Sterilität zur Erzeugung von Hybridsaatgut & nur zur Saatguterzeugung \\
\hline Monsanto & Soja & Herbizid-Toleranz & $\begin{array}{l}\text { nur Import in die EU sowie Lagerung und } \\
\text { Verarbeitung }\end{array}$ \\
\hline Plant Genetic Systems & Raps $^{* *}$ & $\begin{array}{l}\text { Männliche Sterilität zur Erzeugung von Hybridsaatgut } \\
\text { sowie Herbizid-Toleranz }\end{array}$ & - \\
\hline AgrEvo & Mais & Herbizid-Toleranz & - \\
\hline AgrEvo & Raps & Herbizid-Toleranz & $\begin{array}{l}\text { nur Import in die EU sowie Lagerung und } \\
\text { Verarbeitung }\end{array}$ \\
\hline Monsanto] & Mais & Insekten-Resistenz & - \\
\hline Northrup & Mais & Insekten-Resistenz & $\begin{array}{l}\text { nur Import in die EU sowie Lagerung und } \\
\text { Verarbeitung }\end{array}$ \\
\hline Florigene & Nelke* & Veränderung der Blütenfarbe & - \\
\hline Florigene & Nelke & verlängerte Haltbarkeit & - \\
\hline Florigene & Nelke* & Veränderung der Blütenfarbe & - \\
\hline
\end{tabular}

${ }^{*} b z w .{ }^{* *}=$ gentechnisch veränderte Pflanzen, die aus verschiedenen Transformationsexperimenten hervorgegangen sind und deren Inverkehrbringen daher auch separat beantragt werden mussten.

(Stand:Oktober 1999; Quelle: Robert Koch-Institut, Berlin; http://www.fb5.rki.de) 


\section{Originalien und Übersichtsarbeiten}

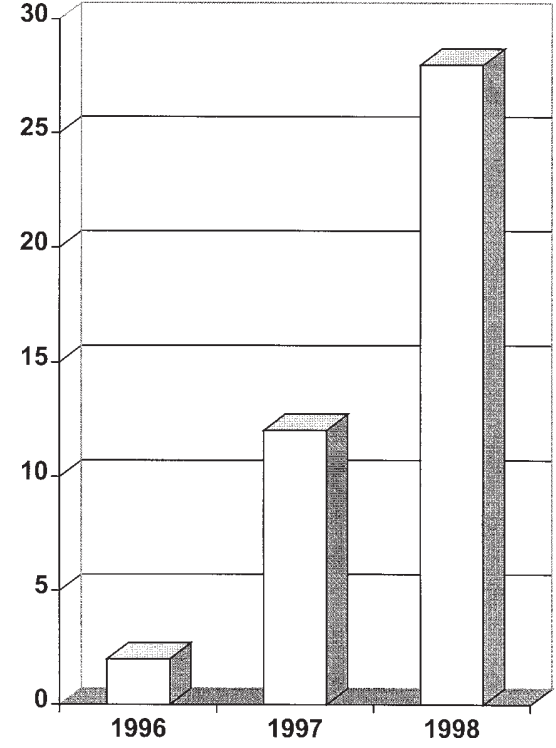

Abb. $2 \Delta$ Weltweite Gesamtfläche (ohne China) für den kommerziellen Anbau von gentechnisch veränderten Pflanzen in den Jahren 1996 bis 1998. Angaben in Mill. Hektar (Quelle: International Service for the Acquisition of Agri-biotech Applications, 1998)

der Wissenschaft ganz überwiegend vertretene Auffassung. Nicht um Erkenntnisse, sondern um - nicht zu berücksichtigende - Spekulationen handelt es sich aber, wenn Aussagen weder einen empirisch belegbaren noch einen wissenschaftlich-theoretisch präzisierbaren Anknüpfungspunkt haben. Risikoerwägungen nach dem Stand der Wissenschaft bedeutet, dass zum Beispiel ein Antrag auf ein Freisetzungsexperiment mit gentechnisch veränderten Pflanzen genehmigt werden muss, wenn sich nach dem Stand der Wissenschaft keine abschlägigen Gründe gegen dieses Freisetzungsexperiment ergeben; es bedeutet aber auch, dass sich grundsätzlich im Verlauf eines bereits genehmigten Freisetzungsexperimentes neue wissenschaftliche Erkenntnisse ergeben können, die eine Neubewertung (und damit möglicherweise auch eine Untersagung) dieses Freisetzungsexperimentes bewirken.

Risikoerwägung nach dem Stand der Wissenschaft berücksichtigt wissenschaftliche Erkenntnisse, nicht aber Spekulationen, deren Aussagen weder empirisch belegbar sind, noch einen wissenschaftlich-theoretisch präzisierbaren Anknüpfungspunkt haben.
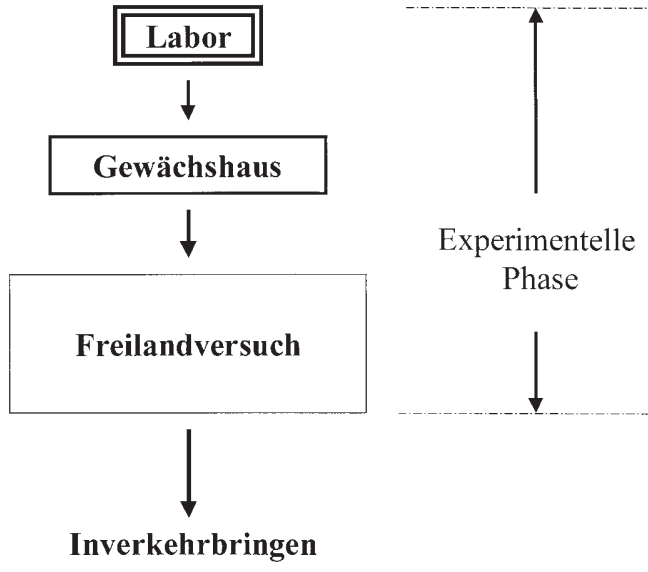

Experimentelle

Phase

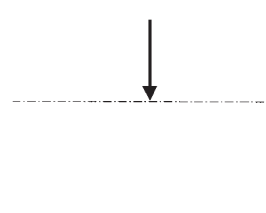

Abb. $3<$ Schematische Darstellung des ,Step-By-Step-Prinzips" von der Transformation einer Pflanze im Labor über die zwei weiteren experimentellen Stadien im Gewächshaus und Freiland bis hin zum Inverkehrbringen.Weitere Erläuterungen im Text
Das Gentechnikrecht gibt vor, in welchem Rahmen Risikoerwägungen zu erfolgen haben (Abb. 4). Die nach $\$ 1$ GenTG vor möglichen Gefahren gentechnischer Verfahren oder Produkte zu schützenden Rechtsgüter sind Leben und Gesundheit von Menschen, Tiere, Pflanzen, Sachgüter und die Umwelt in ihrem Wirkungsgefüge. Zunächst erscheint es ohne weiteres einsichtig, was diese gesetzliche Regelung ausdrückt und erreichen will. Dies mag auch für die fünf zuerst genannten Rechtsgüter so zutreffen, ist aber bei dem Rechtsgut „Umwelt in ihrem Wirkungsgefüge” aus naturwissenschaftlicher Sicht problematisch. Wenn „Umwelt in ihrem Wirkungsgefüge" eine juristische Fassung dessen ist, was generell unter Ökosystem begriffen wird, so darf nicht unberücksichtigt bleiben, dass es ein ökologisches Risiko oder einen ökologischen Schaden per se nicht gibt. Ökologische Untersuchungen allein können niemals die Frage von Schäden in Ökosystemen beantworten, sondern nur das Ausmaß an Veränderungen beschreiben. Es bleibt

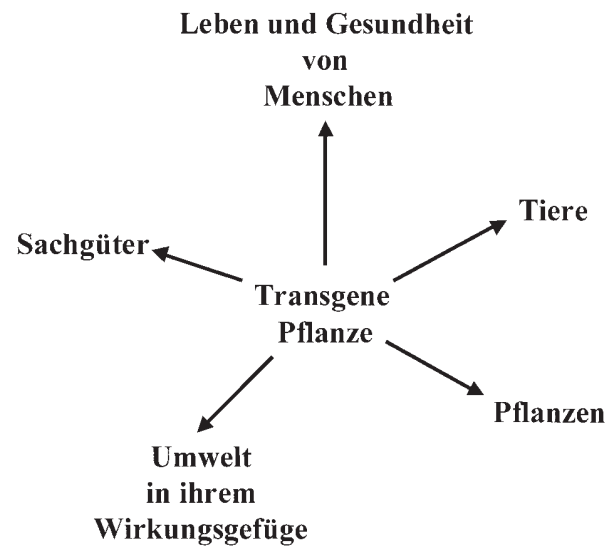

eine gesellschaftliche Entscheidung, ob und was überhaupt als ein Schaden betrachtet wird. Der Rat von Sachverständigen für Umweltfragen definiert zum Beispiel Schäden im ökologischen Sinne als solche Veränderungen, die über das natürliche Schwankungsmaß der betroffenen Populationen oder Ökosysteme hinausgehen und sich oft nur über größere Zeiträume manifestieren sowie Veränderungen, die entweder überhaupt nicht oder oft erst Jahrzehnte nach der menschlichen Einwirkung und mit hohem Aufwand rückgängig gemacht werden können [3]. Mit Blick auf mögliche schädliche Einwirkungen auf das Ökosystem hat es also primär keine große Bedeutung, ob die einzuschätzenden Pflanzen gentechnisch verändert oder nicht gentechnisch verändert sind und ob sie zur einheimischen Flora zählen oder eingeschleppt worden sind. Unerwünschte ökologische Folgen können möglicherweise aus dem Anbau bestimmter transgener Pflanzen ebenso wie aus dem Anbau konventionell gezüchteter Pflanzensorten erwartet werden.
Abb. 4 Inhaltliche Darstellung von §1, 1 . GenTG. Zweck dieses Gesetzes ist es, Leben und Gesundheit von Menschen, Tieren, Pflanzen sowie die sonstige Umwelt in ihrem Wirkungsgefüge und Sachgüter vor möglichen Gefahren gentechnischer Verfahren und Produkte zu schützen und dem Entstehen solcher Gefahren vorzubeugen. Weitere Erläuterungen im Text 


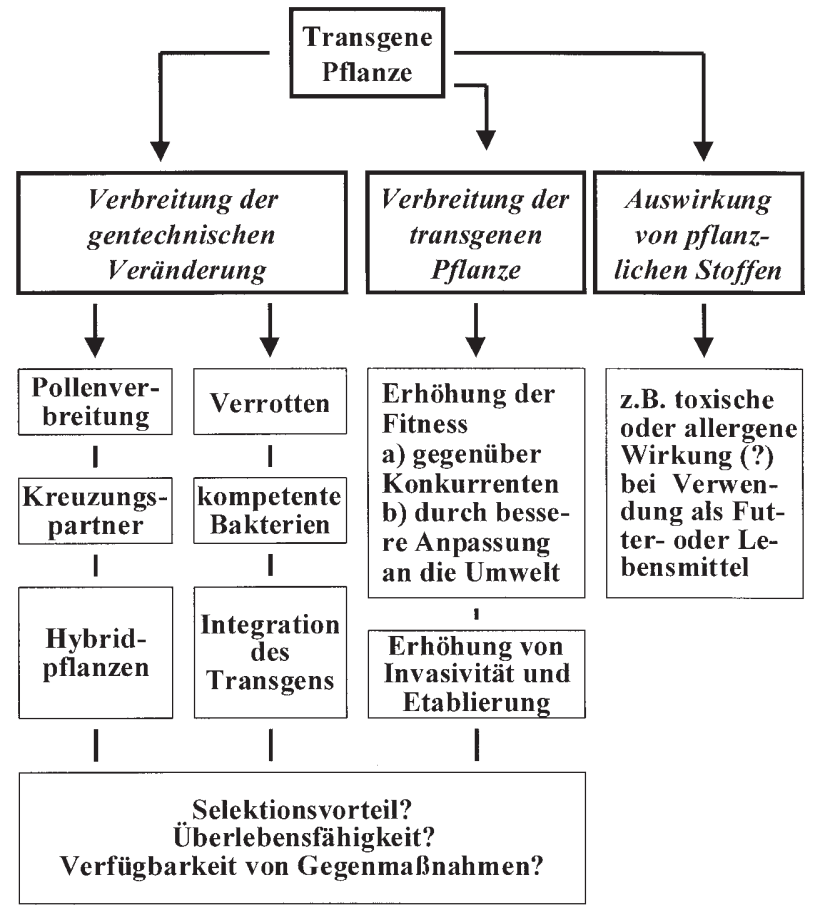

Abb. 5 Schematische Darstellung einiger Aspekte zur Risikoerwägung für Freisetzungsexperimente mit gentechnisch veränderten Pflanzen. Weitere Erläuterungen im Text

\section{Bei der Erwägung potentieller Risiken gentechnisch veränderter Pflanzen sind verschiedene denkbare Auswir- kungen zu berücksichtigen.}

Um mögliche Auswirkungen - und damit auch potentielle Schädigungen - der im $\$ 1$ GenTG benannten Rechtsgüter erden, in welcher Weise gentechnisch veränderte Pflanzen auf diese möglicherweise schädigend einwirken können. Exemplarisch sind drei potentielle Einwirkungswege in dem Schema der Abb. 5 dargestellt. Diese potentiellen Einwirkungswege können die Verbreitung der gentechnischen Veränderung, die VerAuswirkung von pflanzlichen Stoffen bei der Verwendung der transgenen Pflanzen zur Lebens- oder Futtermittelherstellung sein. Dies trifft nicht nur für das Inverkehrbringen, sondern auch bei Freisetzungsvorhaben für die unzulässige Entnahme von transgenen Pflanzen oder Pflanzenteilen für Lebensmitteloder Futtermittelzwecke zu.

Grundsätzlich stellen die angegebenen Einzelschritte innerhalb der drei Rubriken der Abb. 5 jeder für sich noch kein Risiko und damit auch keine Gekennen zu können, muss geprüft werbreitung der transgenen Pflanze und die fährdung der zuvor genannten Rechtsgüter dar, wenn dies auch oft in der Öfspiel ist die Ausbreitung von transgenem Pollen von einem Freisetzungsstandort mitnichten schon eine Gefährdung. Es müssen z.B. weitere Schritte, wie das Vorhandensein von kompatiblen Kreuzungspartnern, die tatsächliche Befruchtung, die Enstehung von Hybridsamen, daraus lebensfähige Hybridpflanzen, die ihrerseits wieder zur Samenbildung befähigt sein müssen, erfolgen, damit erwogen werden kann, ob z.B. von derartigen Hybridpflanzen unter den Bedingungen am Freisetzungsstandort und in seiner weiteren Umgebung aufgrund der hinzugewonnenen gentechnischen Veränderung zu erwarten ist, dass sie einen Selektionsvorteil oder eine größere Überlebensfähigkeit haben. Aber selbst dann ist noch zu prüfen, ob ihrem etwaigen Auftreten nicht mit herkömmlichen erprobten landwirtschaftlichen Methoden begegnet werden kann. Die gleiche fentlichkeit behauptet wird. Zum Bei-

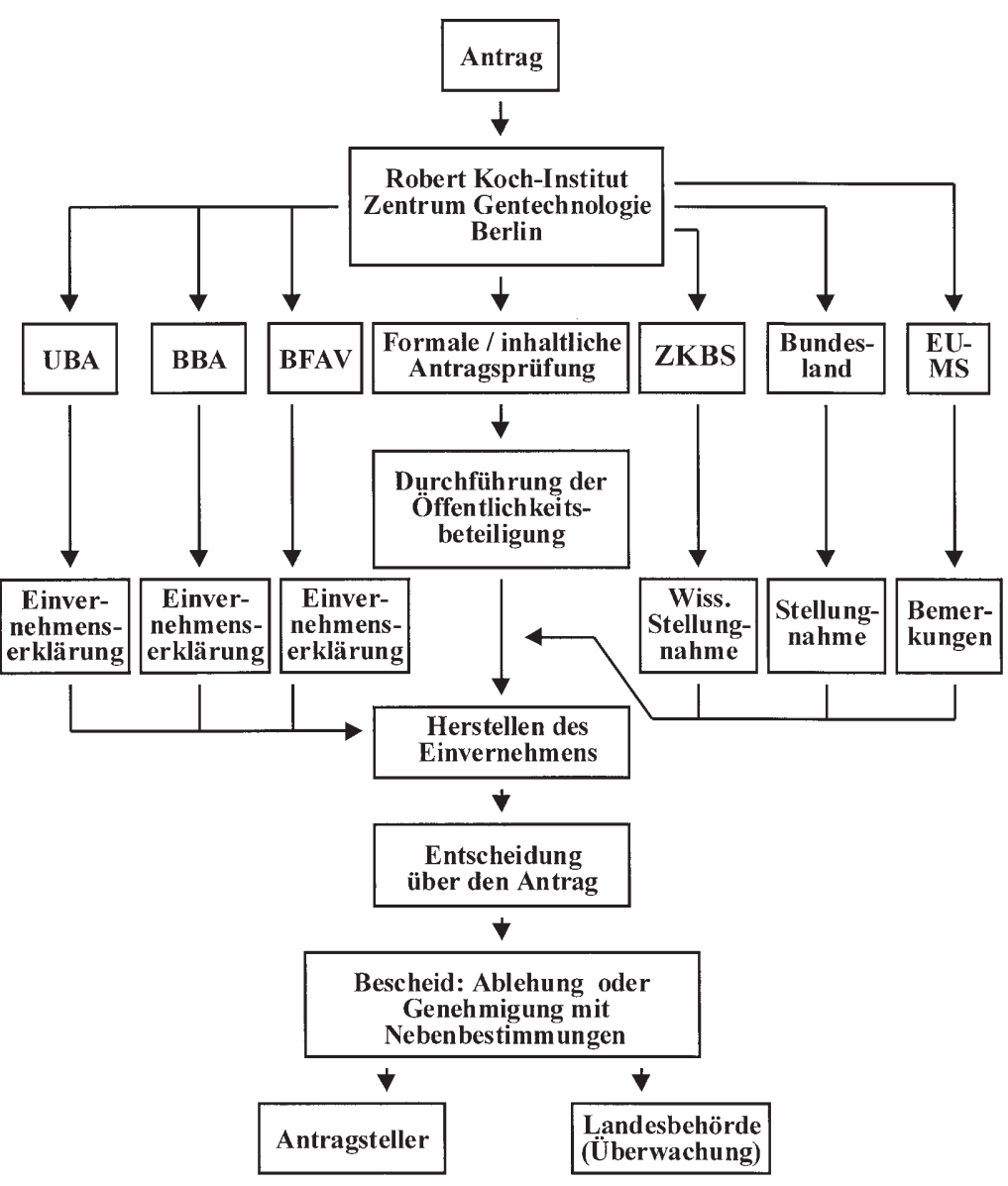

Abb. $6 \Delta$ Schematische Darstellung des Verfahrens und der daran beteiligten Institutionen für Anträge auf Freisetzungsversuche mit gentechnisch veränderten Pflanzen. Weitere Erläuterungen im Text 
Fragestellung ergibt sich natürlich, wenn die transgenen Pflanzen selbst eine erhöhte Fitness aufweisen sollten und damit prädestiniert wären für eine erhöhte Invasivität und Etablierung im umgebenden Ökosystem.

Die zweite Variante der denkbaren Verbreitung der gentechnischen Veränderung verläuft über den sogenannten Horizontalen Gentransfer, also dem Übergang des Transgens von der transgenen Pflanze auf Bakterien. Dies könnte theoretisch einerseits über verrottendes transgenes Pflanzenmaterial im Boden oder auch im Zuge der Verdauung von transgenem Pflanzenmaterial im Magen-Darmtrakt erfolgen. Es ist anzumerken, dass es bislang nicht geglückt ist, diesen Horizontalen Gentransfer im Freiland unter natürlichen Bedingungen überhaupt nachzuweisen, was dazu führt, dass man ihn als zumindest äuBerst seltenes Ereignis einstuft. Entsteht daraus nun ein Dilemma für die Risikoerwägungen? Dies ist nicht der Fall, wenn man davon ausgeht, dass ein solcher Horizontaler Gentransfer möglich wäre und dass unter dieser theoretischen Voraussetzung bewertet wird, welche Vorteile ein Bakterium durch die funktionale Integration des Transgens aus der Pflanze erlangen und wie solchen modifizierten Mikroorganismen methodisch begegnet werden könnte.

Das in der Abb. 5 dargestellte Schema gibt nur beispielhaft einen Teil der Risikoerwägungen wieder, die für die Genehmigung von Anträgen auf Freisetzung von gentechnisch veränderten Pflanzen von Bedeutung sein können. In Deutschland ist das Robert Koch-Institut als Zulassungsbehörde zuständig für derartige Anträge; die Genehmigungsverfahren beziehen als Einvernehmensbehörden das Umweltbundesamt (UBA) und die Biologische Bundesanstalt für Landwirtschaft und Forsten (BBA) (gegebenenfalls auch die Bundesforschungsanstalt für Viruskrankheiten der Tiere (BFAV)) mit ein; außerdem sind die Zentrale Kommission für die Biologische Sicherheit (ZKBS), das Bundesland, in dem der zukünftige Freisetzungsstandort liegen soll, und die EU-
Mitgliedstaaten (EU-MS) an dem Genehmigungsverfahren beteiligt (Abb.6). Während des Genehmigungsverfahrens erfolgt eine Öffentlichkeitsbeteilung (weitere Erläuterungen siehe [2]).

\section{Im Rahmen bisheriger Genehmigungs- verfahren sind noch keine Gefahren durch freizusetzende transgene Pflan- zen festgestellt worden - derartige $\mathrm{Ge}$ - fahren sind aber bei zukünftigen Frei- setzungsanträgen auszuschließen.}

Bislang haben die im Rahmen der Genehmigungsverfahren für geplante Freisetzungsvorhaben durchgeführten Risikoerwägungen in keinem Fall ergeben, dass zu erwarten gewesen wäre, dass von den freizusetzenden transgenen Pflanzen Gefahren für die zuvor benannten Rechtsgüter ausgehen werden. Mustert man allerdings die wissenschaftlichen Publikationen daraufhin, mit welchen gentechnisch veränderten Pflanzen in Zukunft gerechnet werden muss, so ist nicht mehr auszuschließen, dass einige von ihnen mit einem derartigen Gefahrenpotential belastet sein könnten. Für diese Fälle sieht das Gentechnikgesetz eine Abwägung zwischen den Risiken und dem Nutzen der jeweiligen transgenen Pflanzen vor. Es ist selbstverständlich, dass es keinen generellen Verfahrenskatalog oder Wertmaßstab für derartige Risiko-Nutzen-Abwägungen geben kann; auch dieses Verfahren kann nur in zukünftigen Einzelfällen entwickelt und zu einer Entscheidung gebracht werden. Es sei außerdem daran erinnert, dass eine solche Risiko-Nutzen-Abwägung kein Sonderfall der Gentechnik ist, sondern schon in anderer Weise im medizinischen und pharmazeutischen Bereich praktiziert wird.

$\mathrm{Zu}$ gegebener Zeit wird über solche Risiko-Nutzen-Abwägungen aus dem Bereich der „Grünen Gentechnik” berichtet werden.

\section{Literatur}

1. Brandt $P$ (1997) Zukunft der Gentechnik. Basel:290, Birkhäuser

2. Brandt $P$ (1995) Transgene Pflanzen. Herstellung, Anwendung, Risiken und Richtlinien. Basel:306, Birkhäuser

3. Rat der Sachverständigen für Umweltfragen (1995) Altlasten II. Sondergutachten, Stuttgart
In den vergangenen Wochen erreichten uns die unten aufgeführten Neuankündigungen. Ausgewählte Titel werden in nächster Zeit besprochen.

Hrsg.:D. Reinhardt

Asthma bronchiale im Kindesalter

3., aktual. u. erw. Aufl.; Berlin, Heidelberg, New York:Springer, 1999. 323 S., 82 Abb., 59 Tab., (ISBN 3-540-65240-X), geb., DM 79,-

E. Raith, P. Frank, G. Freundl Natürliche Familienplanung heute

3., überarb. u. erw. Aufl.; Berlin, Heidelberg, New York:Springer, 1999.237 S., 62 Abb., 10 Tab., (ISBN 3-540-65744-4), brosch., DM 49,90

D. Adam. H. Lode

Atemwegsinfektionen

Stuttgart, New York: Thieme, 1999. 190 S., 5 Abb., 44 Tab., (ISBN 3-13-105281-3/694), Tb., DM 39,90

D. Reinhardt et al.

Leitlinien der Kinderheilkunde und Jugendmedizin

München: Urban \& Fischer, 1999. 1000 S., ca. 27 Abb., 132 Tab., (ISBN 3-437-22060-8), Loseblatt Grundwerk, DM 198,- 